\title{
Use of antidiuretic hormone antagonists results in lower hospital resource usage: a retrospective cohort study
}

\author{
Paul Grant', Clare Jamookeeah², Penny Dhanjaß", Gethin Griffith ${ }^{2}$
}

'Oxford Centre for Diabetes, Endocrinology \& Metabolism, Oxford, UK; ${ }^{2}$ Otsuka Pharmaceutical Europe Ltd, Wexham, UK ${ }^{3}$ Otsuka Pharmaceutical UK Ltd, Wexham, UK

\section{Introduction}

- Hyponatraemia is the electrolyte disturbance most commonly encountered in clinical practice (affecting 10-30\% of hospitalised patients) and can be a marker of morbidity in many contexts, increasing mortality rates regardless of cause.

- The syndrome of inappropriate antidiuretic hormone secretion (SIADH) is the most common cause of hypotonic hyponatraemia, ${ }^{2}$ responsible for approximately $30 \%$ of all patients with hyponatraemia.

- Data from the US suggest that patients with hyponatraemia have a greater overall consumption of healthcare resources, and that hyponatraemia is associated with an increased length of hospital stay, ${ }^{3-5}$ and increased direct medical costs. ${ }^{6}$

- The overall annual cost of hyponatraemia in the US alone has been estimated at \$1.6-3.6 billion $^{7}$ - there are no comparable cost data for the UK

- The UK Consensus Statement recommends pharmacological therapy (demeclocycline or tolvaptan) for patients with mild/moderate hyponatraemia secondary to SIADH without severe symptoms, after fluid restriction has been attempted or deemed inappropriate. ${ }^{8}$

- The aim of this study was to understand how hyponatraemia secondary to SIADH is currently treated in England, and to understand the associated resource use.

\section{Methods}

- A retrospective analysis of a patient cohort identified in IMS Hospital Treatment Insights $(\mathrm{HTI})$ through hospital admission diagnosis codes from 1 January 2010 through to 31 October 2013 was conducted.

- The HTI database, comprising details of all admissions and outpatient appointments to NHS hospitals in England, contains records on 3.3 million English patients from a source population of 13 million.

- Adult patients were included if they had a new World Health Organization (WHO) ICD-10 diagnostic code for 'Hypo-osmolality and hyponatraemia' (E87.1) and/or a diagnostic code for SIADH (E22.2) during the study period. - Exclusion criteria were patients $<18$ years of age or with no age recorded, or co-morbid conditions associated with hyponatraemia: liver cirrhosis; liver disease with ascites; heart failure; unspecified adrenocortical insufficiency; hypothyroidism; and type 1 diabetes.

- Study outcomes were captured through relevant WHO ICD-10 codes and comprised hospital admissions, outpatient visits, length of hospital stay, and in-hospital mortality.

\section{Results}

- In total, 37,425 patients were given a clinical code for SIADH or hyponatraemia (Figure 1); $>60 \%$ of patients were $\geq 68$ years of age.

- Of the patients with a diagnosis of hyponatraemia and/or SIADH, 1.8\% were given demeclocycline, $0.3 \%$ were given tolvaptan, and $97.9 \%$ were not given any recorded pharmacological treatment. When pharmacological treatment was given, it was usually as monotherapy.

- Mean time from diagnosis to initiation of therapy was similar for both demeclocycline and tolvaptan (10.2 days).

- Median time was 3 days longer with tolvaptan at 8 days

- Patients receiving tolvaptan had a shorter mean (11.1 vs 19.3 days) and median (10 vs 14 days) length of hospital stay than those given demeclocycline.

- Time to readmission and readmission rates at 30 and 60 days appeared similar between the two treatment groups (Table 1).
Figure 1. Patient population

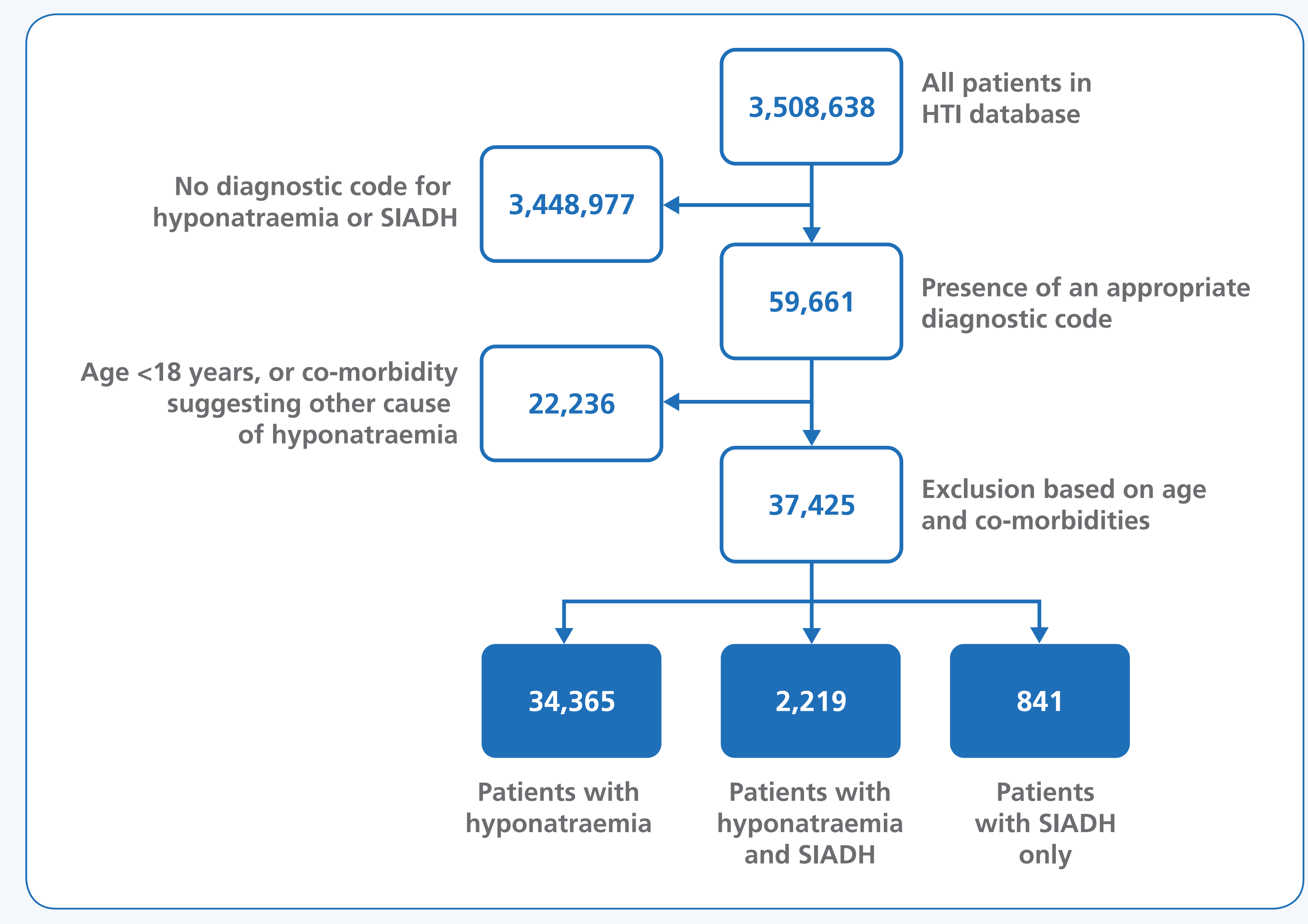

HTI, Hospital Treatment Insights; SIADH, syndrome of inappropriate antidiuretic hormone secretion

Table 1. Patient population

\begin{tabular}{|l|c|c|}
\hline & Demeclocycline & Tolvaptan \\
\hline Time to re-admission, days & & \\
N (hospitalisations) & 437 & 61 \\
Mean & 45.6 & 26.5 \\
Median & 5 & 14 \\
Range & $0-1041$ & $0-176$ \\
\hline Re-admission at $\mathbf{3 0}$ days, \% (n) & $74.6(25.4)$ & $72.1(44)$ \\
\hline Re-admission at $\mathbf{6 0}$ days, \% (n) & $82.8(362)$ & $85.2(52)$ \\
\hline
\end{tabular}

SIADH, syndrome of inappropriate antidiuretic hormone secretion

- Tolvaptan treatment was associated with a 29\% fewer accident and emergency (A\&E) attendances per patient than demeclocycline $(p=0.041)$

- Number of A\&E attendances was significantly reduced across both treatments compared with patients not receiving any pharmacological treatment $(p<0.00005)$

- Receiving pharmacological treatment was associated with significant reductions in the number of outpatient appointments per patient

- There were $0.788,0.693$ and 0.540 appointments/patient for demeclocycline, tolvaptan and no pharmacological treatment, respectively $(p<0.05)$

\section{Conclusions}

- Hyponatraemia and SIADH represent a substantial healthcare burden, and there are considerable variations in assessment and treatment.

- Under-reporting of hyponatraemia secondary to SIADH presents potentially serious implications for hospital remuneration.

- Pharmacological treatment results in considerably fewer A\&E attendances than with no treatment; effective control of hyponatraemia may result in lower resource usage.

- Management of SIADH with tolvaptan may result in lower resource usage for hospitals and the wider health economy versus treatment with demeclocycline. 